This item was submitted to Loughborough's Research Repository by the author.

Items in Figshare are protected by copyright, with all rights reserved, unless otherwise indicated.

\title{
Real time detection of low adhesion in the wheel/rail contact
}

PLEASE CITE THE PUBLISHED VERSION

https://doi.org/10.1177/0954409713503634

\section{PUBLISHER}

(C) IMechE. Published by Sage

\section{VERSION}

AM (Accepted Manuscript)

\section{PUBLISHER STATEMENT}

This work is made available according to the conditions of the Creative Commons Attribution-NonCommercialNoDerivatives 4.0 International (CC BY-NC-ND 4.0) licence. Full details of this licence are available at: https://creativecommons.org/licenses/by-nc-nd/4.0/

\section{LICENCE}

CC BY-NC-ND 4.0

\section{REPOSITORY RECORD}

Hubbard, Peter D., Christopher P. Ward, Roger Dixon, and Roger M. Goodall. 2019. "Real Time Detection of Low Adhesion in the Wheel/rail Contact". figshare. https://hdl.handle.net/2134/35041. 
Journal name

\section{Real Time Detection of Low}

\section{Adhesion in the Wheel/Rail Contact}

():-

CThe Author(s) 2010

Reprints and permission:

sagepub.co.uk/journalsPermissions.nav

DOI: $10.1177 / 1081286510367554$

http://mms.sagepub.com

Peter Hubbard, Chris Ward, Roger Dixon, Roger Goodall

School of Electronic, Electrical and Systems Engineering,

Loughborough University,

Loughborough,

LE11 3TU

United Kingdom

(e-mail: P.D.Hubbard3@ @lboro.ac.uk)

Abstract 
Condition monitoring of railway vehicles has been highlighted by the railway industry as a key enabling technology for future system development. The primary uses for this could be the improvement of maintenance procedures and/or the identification of high risk vehicle running conditions. Advanced processing of signals means these tasks could be accomplished without the use of cost prohibitive sensors.

This paper presents a system for the on-board detection of low adhesion conditions during the normal operation of a railway vehicle. Two different processing methods are introduced. The first method is a modelbased approach that uses a Kalman-Bucy filter to estimate creep forces, with subsequent post processing for interpretation in to adhesion levels. The second non model-based method targets the assessment of relationships between vehicle dynamic responses to observe any behavioural differences as a result of an adhesion level change.

Both methods are evaluated in specific case studies using a British Rail (BR) Mark 3 coach, inclusive of a BR BT-10 bogie, and a generic modern passenger vehicle based on a contemporary bogie design. These vehicles were chosen as typical application opportunities within the UK.

The results are validated with data generated by the multi-body simulation software VAMPIRE ${ }^{\circledR}$ for realistic data inputs, representing a key scientific achievement.

Keywords

Railways, Vehicle Dynamics, Kalman Filters, Low Adhesion

\section{Introduction}

Low adhesion or the 'leaves on the line' problem is a large issue often misunderstood by the industry and general public alike. There is currently a lack of information about the changing picture of areas of low 
adhesion with respect to short term trends (over a daily period), and macro trends (across the seasons). The generally established methods of identifying these areas of low adhesion involve mapping activations of wheel slide and wheel slip protection systems to track locations. This represents a blind-spot in awareness as adhesion cannot be assessed outside of braking or traction zones, potentially resulting in application of costly mitigation methods such as railhead cleaning and timetable changes where none are needed.

The research described in this article has been performed as part of a project commissioned by RSSB in the UK and supported by the TSLG (Technology Strategy Leadership Group) to investigate methods of detecting low adhesion. This research is a progression from a feasibility study, also commissioned by RSSB, to investigate advanced methods of detecting areas of low adhesion in real time using modest cost inertial sensors mounted to in-service vehicles. This study demonstrated that the motions of a railway vehicle (in both lateral and yaw directions) vary as the adhesion conditions at the wheel/rail interface change (1). Fundamentally, this means that if the changes in the running dynamics as a result of low adhesion can be observed and understood, the adhesion at all points across a rail network can be inferred during normal running, i.e. not only when slip/slide events are triggered.

A number of methods have been proposed to analyse data provided by inertial sensors mounted on the wheelsets, bogie and vehicle body. The main focus of the research has been developing a model-based approach $(2 ; 3)$ that uses a fundamental understanding of the physics of a rail vehicle to estimate wheel-rail creep forces that can not be measured directly. The application of this technique, including comparison work and analysis of data produced by a high fidelity railway vehicle dynamics simulation, will be presented in Section 2. 
The other stream of work highlighted in this paper is the direct analysis of measured data in order to identify any features in the relationships between component dynamic behavious as adhesion levels change. The current findings of this work are presented in Section 3.

\section{Model-based Estimator}

The concept of using a model-based estimator to identify faults and failures or estimate systems parameters is widely suggested in railway specific applications. There are examples of suspension parameter modelling (4; 5), suspension fault detection (6; 7), suspension condition monitoring (8) and wheel/rail condition monitoring (9). The opportunities are broad for this style of investigation with some publications including numerous different examples of fault identification or condition monitoring with model based methods $(10 ; 11 ; 12)$.

In a similar fashion to the model-based estimation techniques highlighted above, the concept of using such an approach to approximate adhesion levels whithin the wheel/rail interface has been developed and tested against linear suspension models with complex, non-linear wheel/rail interface mechanics generated in MATLAB/Simulink $(1 ; 9)$. The work presented in this article extends this previous study by applying the creep force estimation method to more representative simulation data. This data was supplied in the form of outputs taken from the multi-body physics software (MBS) package VAMPIRE ${ }^{\circledR}$, generated for this project by DeltaRail (13). VAMPIRE ${ }^{\circledR}$ is a well validated, specialist rail vehicle dynamics modelling software that simulates multi-degree of freedom/multi-bodied interactions of a railway vehicle.

MBS packages (such as VAMPIRE ${ }^{\circledR}$ ) are currently the closest means of generating vehicle dynamic data short of the costly process of full scale testing. In this study, data produced from the MBS is treated as a close representation of the data outputs from real accelerometers and yaw rate gyros mounted on the 
wheelset, bogies and body of railway vehicles. The added advantage from a MBS package is that the creep forces are also available, enabling high fidelity validation of the estimation method.

By using VAMPIRE ${ }^{\circledR}$ simulation data generated, it is possible to treat the data outputs as sensor readings in order to verify any model based estimators created. The outputs from these can then be compared against those that are captured directly from VAMPIRE ${ }^{\circledR}$. This use of MBS data is considered as an intermediate step to prove the efficacy of the methods before the final validation occurs through full scale testing.

This section gives a brief overview to the generation of a model based estimator and presents the results when this estimator is applied to VAMPIRE ${ }^{\circledR}$ test data.

\subsection{Methodology}

The model-based estimator approach used previously is based around the well-known Kalman-Bucy filter (14). In this method the filter requires a well validated, linearised plan view model of the vehicle suspension system in order to estimate the total lateral force and yaw moments at the wheel/rail interface $(3 ; 9)$. It has been shown $(15 ; 16)$ that the yaw and lateral dynamic responses contain the dominant characteristics of the motion when the adhesion levels are modified and that the other system dynamic responses can be neglected due to their secondary importance.

The first step of the process is to create and validate a second representative simulation model of the system in question in MATLAB/Simulink. The linearised suspension component from this model can then be converted into state space form and used to form a Kalman-Bucy filter. 


\subsection{Model Development and Validation}

The vehicle chosen for this case study is a generic modern passenger, Diesel Multiple Unit (DMU) vehicle, that is based loosely on a British class 158 vehicle. This vehicle represents a typical example of a vehicle likely to benefit from on-board adhesion detection.

Figure 1 shows a simplified plan-view linear representation of a railway vehicle primary suspension. A set of linear equations to describe the dynamics is derived from this geometry, by considering the force/moment balance equations around the wheelset. The parameters used are defined in table 1.

\begin{tabular}{cc} 
& Calibration Data \\
\hline Parameter & Description \\
\hline \hline$y_{F F}$ & wheelset lateral position \\
$\psi_{F F}$ & wheelset yaw position \\
$y_{B}$ & bogie lateral position \\
$\psi_{B}$ & bogie yaw position \\
$k_{x}$ & primary spring stiffness (longitudinal) \\
$k_{y}$ & primary spring stiffness (lateral) \\
$k_{b x}$ & bush spring stiffness (longitudinal) \\
$k_{b y}$ & bush spring stiffness (lateral) \\
$l_{\text {axlebox }}$ & axlebox semi-spacing \\
$l_{b u s h b o x}$ & radius arm length \\
$l_{w h l s e t}$ & wheelset semi-spacing \\
$F_{R y / L y}$ & creep forces at the right and left wheel/rail interface (lateral) \\
$F_{R x / L x}$ & creep forces at the right and left wheel/rail interface (longitudinal) \\
$F_{g}$ & gravitational stiffness' - resolved normal force due to weight and contact angle \\
$M_{g}$ & gravitational stiffness' - resolved moment due to weight and contact angle \\
\hline
\end{tabular}

Table 1. Parameters used for the primary suspension dynamic model

The linear and yaw dynamics of the wheelset are therefore defined by:

$$
\begin{aligned}
m \ddot{y}_{F F} & =F_{R y}+F_{L y}+F_{g}+2 k_{y}\left(\left(y_{b}+\psi_{b} l_{b u s h}\right)-y_{F F}\right) \\
& +2 k_{b y}\left(\left(y_{b}+\psi_{b}\left(l_{\text {whlset }}-l_{\text {bush }}\right)\right)-\left(y_{F F}+\psi_{F F} l_{b u s h}\right)\right)
\end{aligned}
$$




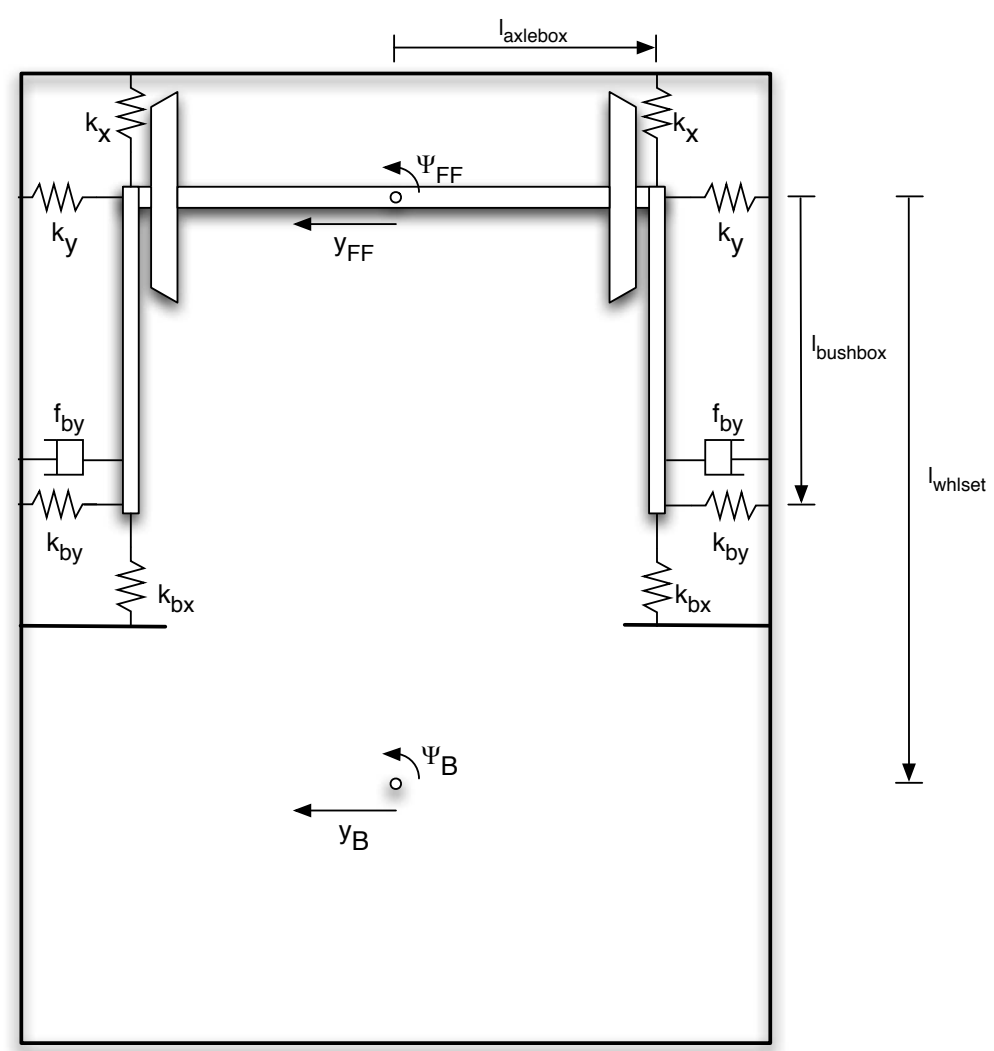

Fig. 1. Plan-view, linear model of the Modern Vehicle primary suspension system

$$
\begin{aligned}
I_{w x} \ddot{\psi}_{F F} & =R_{R y} F_{R x}-R_{L y} F_{L x}+M_{g} \\
& +2 k_{b y}\left(\left(y_{b}+\psi_{b}\left(l_{w h l s e t}-l_{b u s h}\right)\right)-\left(y_{F F}+\psi_{F F} l_{b u s h}\right)\right) l_{b u s h} \\
& +2\left(k_{x}+k_{b x}\right)\left(\psi_{b}-\psi_{F F}\right) l_{\text {axlebox }}^{2}
\end{aligned}
$$

where minor terms have been neglected. 
The equation set generated was used to form a dynamic linear model in a MATLAB/Simulink environment. The model generated contains two selectable contact force descriptions based on the works of Kalker and Polach $(17 ; 18)$. In addition, a VAMPIRE ${ }^{\circledR}$ test case, against which this Simulink model is to be validated, was generated whereby a whole vehicle model virtually travelled along a length of perfect track containing a single $5 \mathrm{~mm}$ lateral step after 1 second of travel.

A useful test to validate the linear model is to check that calculated component accelerations, as defined in equations (2.1) and (2.2), are close to the equivalent accelerations recorded in the VAMPIRE ${ }^{\circledR}$ simulations. This would confirm that the set of equations formed by the consideration of the force/moment balance of the suspension components in a linear, plan-view sense sufficiently match the suspension forces experienced in the VAMPIRE ${ }^{\circledR}$ simulation. To perform this, the contact forces, bogie and wheelset dynamic responses recorded in a VAMPIRE ${ }^{\circledR}$ simulation are input into the linear Simulink model which in turn derives the total wheelset lateral and yaw accelerations based on the resultant suspension loading.

Figure 2 shows the so called 'open loop' response of the modern vehicle simulation model in dry conditions and compares the output to the values simulated by VAMPIRE ${ }^{\circledR}$. The solid blue lines show the resultant derived wheelset accelerations in the lateral and yaw directions from the linear Simulink model. The green dashed line shows the acceleration values recorded in VAMPIRE ${ }^{\circledR}$. It can be seen that the matches are close, but have a number of discrepancies that are a result of differences in the Force/Moment balance equations. These differences between the models can be accounted for by:

- The 3D suspension geometry of VAMPIRE ${ }^{\circledR}$ compared to the 2D plan view assumption of the Simulink model. 

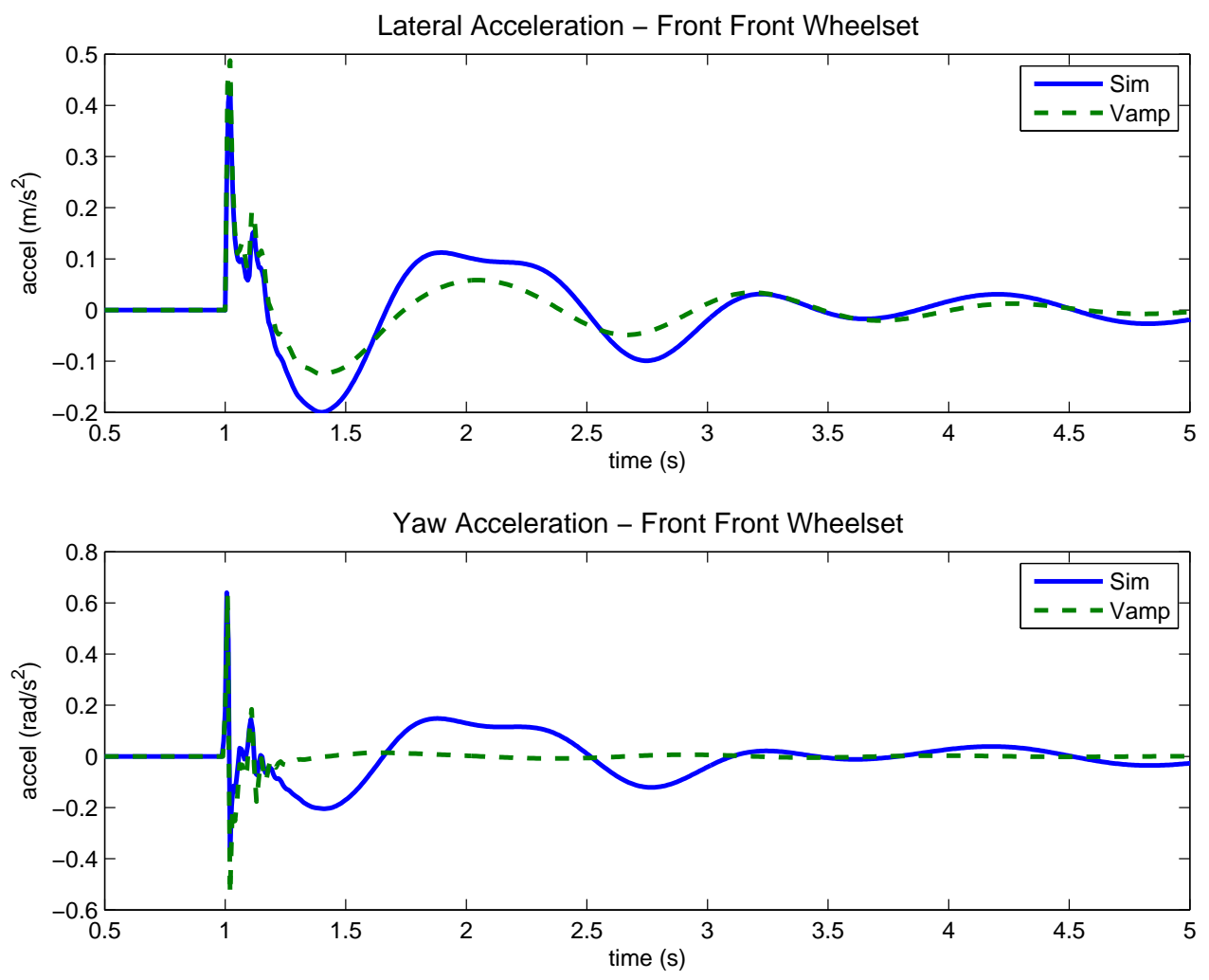

Fig. 2. Figures showing the derived values of lateral and yaw acceleration overlaying the recorded VAMPIRE ${ }^{\circledR}$ values. These are taken from the centre of mass of the front wheelset of the front bogie - hence the 'front front' label.

- Linearisation of components in the Simulink model compared to non-linear components of the VAMPIRE $^{\circledR}$ model.

- Simplification of some suspension components in the Simulink model.

In terms of application to a Kalman-Bucy filter, the differences shown above are small enough to allow the linear model to form the basis of the estimator. 


\subsection{Creep Force Estimation Technique}

The linear suspension model generated for the modern passenger vehicle is converted into standard state space form given by the state and output equations:

$$
\begin{aligned}
& \dot{\mathbf{x}}=\mathbf{A}_{\mathbf{k}} \mathbf{x}+\mathbf{B}_{\mathbf{k}} \mathbf{u} \\
& \mathbf{y}=\mathbf{C}_{\mathbf{k}} \mathbf{x}+\mathbf{D}_{\mathbf{k}} \mathbf{u}
\end{aligned}
$$

Previous work has shown (9) that the Kalman-Bucy filter cannot distinguish between the creep forces and the resolved normal forces as separate states and therefore are combined into one state, as defined by:

$$
\begin{gathered}
F_{F F}=F_{L y}+F_{R y}+F_{g} \\
M_{F F}=R_{R y} F_{R x}-R_{L y} F_{L x}+M_{g}
\end{gathered}
$$

The state space model is formed by choosing the states to be the position and velocity of the wheelset (in both lateral and yaw directions) along with the contact forces:

$$
\mathbf{x}=\left[y_{F F}, \dot{y}_{F F}, \psi_{F F}, \dot{\psi}_{F F}, F_{F F}, M_{F F}\right]^{T}
$$

The bogie position and velocity (in both lateral and yaw directions) are included here in the input vector u, where:

$$
\mathbf{u}=\left[y_{B F}, \dot{y}_{B F}, \psi_{B F}, \dot{\psi}_{B F}\right]^{T}
$$


The state equation (2.3) can now be satisfied by using equations (2.1) and (2.2) to populate the matrices $\mathbf{A}_{\mathbf{K}}$ and $\mathbf{B}_{\mathbf{K}}$.

The Kalman-Bucy filter is formed from the equations:

$$
\begin{gathered}
\dot{\hat{\mathbf{x}}}=\mathbf{A}_{\mathbf{k}} \hat{\mathbf{x}}+\mathbf{B}_{\mathbf{k}} \mathbf{u}+\mathbf{K}(\mathbf{y}-\hat{\mathbf{y}}) \\
\hat{\mathbf{y}}=\mathbf{C}_{\mathbf{k}} \hat{\mathbf{x}}
\end{gathered}
$$

The derivative estimated states $\dot{\hat{\mathbf{x}}}$ are formed from two parts; the expected value, based on the suspension description defined as a function of $\mathrm{Ak}, \mathrm{Bk}$, the current state estimate, $\hat{\mathbf{x}}$, and the current inputs, $\mathbf{u}$, and a correction factor based on a scaled difference between sensor measurements $\mathbf{y}$ and expected sensor outputs $\hat{\mathbf{y}}$. In this case, the values of $\hat{\mathbf{y}}$ are the estimated states (defined by choosing $\mathbf{C}_{\mathbf{k}}$ in equation (2.10) accordingly) and $\mathbf{y}$ represents the actual sensor values captured from a vehicle. In this configuration the measurements required would be the leading wheelset and bogie positions and velocities, in both the lateral and yaw directions. These could be derived from accelerometers or yaw gyros to reduced measurement complexity.

The Kalman gain, $\mathbf{K}$, is derived by identifying a degree of certainty with each of the state models (9). By setting the creep force state models as highly uncertain compared to the vehicle dynamics state models, the filter can be used to approximate the creep forces by extracting them from the estimated state vector $\hat{\mathbf{x}}$.

Figure 3 shows an evaluation of this method at two different levels of adhesion; ideal dry conditions and low levels of adhesion. The actual values of friction defined for these levels were chosen to represent different adhesion conditions at which the vehicle operates, dry $(\mu=0.56)$ and low $(\mu=0.072)$. 

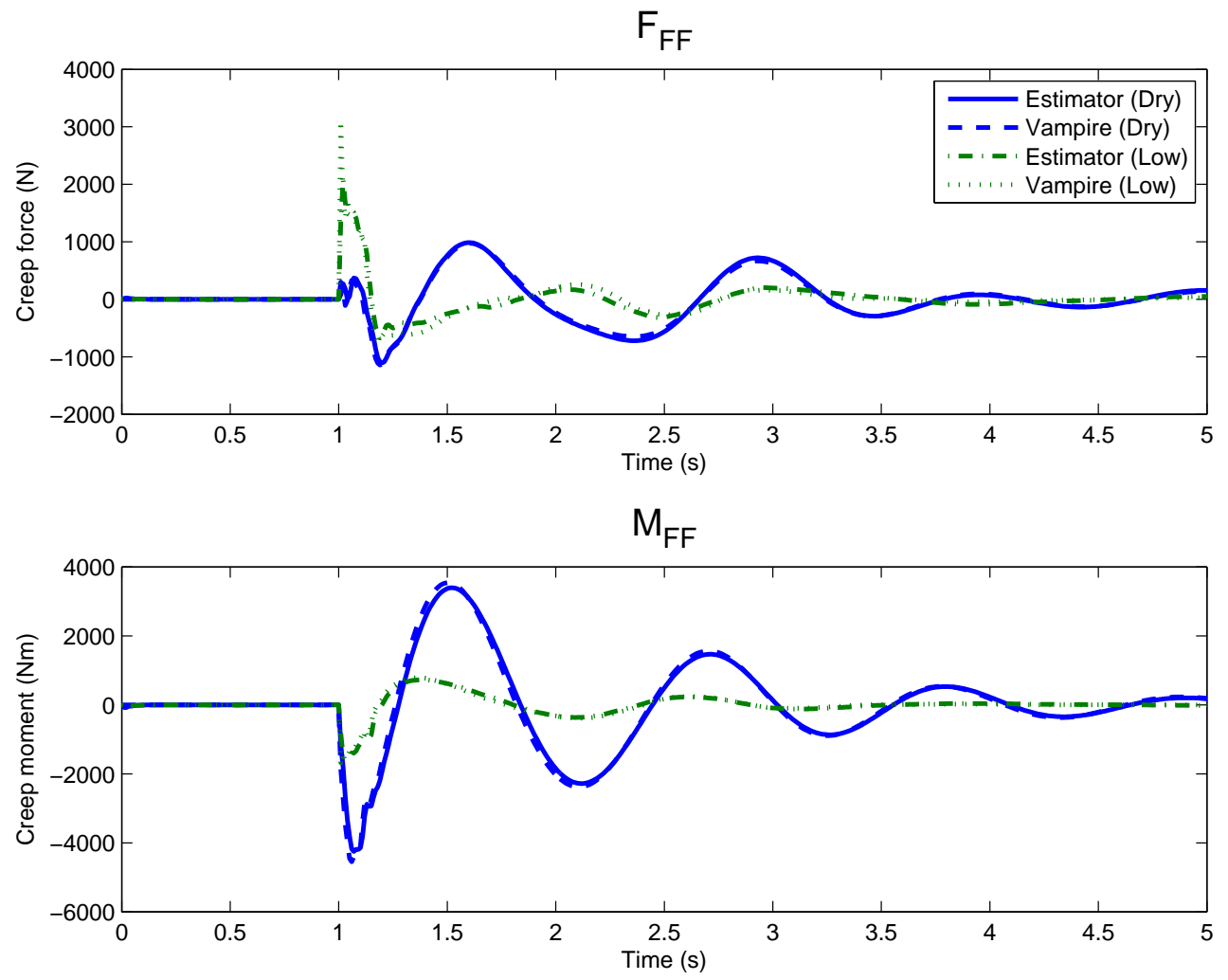

Fig. 3. Figure showing the estimated lateral creep force and estimated total creep moment, overlaying the recorded values.

In each case, VAMPIRE ${ }^{\circledR}$ data was generated using the same section of track as in section 2.2. Selected states from this are then passed into the filter representing equivalent sensor data measured in real time.

It can be seen that the outputs of the model-based estimator demonstrate good agreement to the simulated lateral contact forces and creep moments at both levels of adhesion. This confirms that although the linear approximations demonstrated in Figure 2 were not exact, they were good enough to facilitate the implementation of the creep force estimation. 


\subsection{Verification with VAMPIRE ${ }^{\circledR}$}

A number of simulations were produced in VAMPIRE ${ }^{\circledR}$ in order to validate the capability of the KalmanBucy filter in more demanding scenarios. In these simulations, the VAMPIRE ${ }^{\circledR}$ model of the modern DMU virtually travelled a typical section of track under different operating conditions. For the results presented in this section, the track used contained irregularities (for a 200kph track) in both lateral and vertical directions, as opposed to the previous test where there existed only a single, lateral step input. These irregularities are statistically similar to those of a typical high speed line found in the UK. The vehicle travelled for 60 seconds at full line speed (defined here as 200kph) and the positions, velocites and accelerations of the vehicle components (wheelsets, bogies and vehicle body) along with the contact forces experienced were recorded. As before, this allowed the Kalman-Bucy filter to use the recorded quantities from VAMPIRE ${ }^{\circledR}$ as if they were sensor readings taken from the vehicle. This test was repeated at three different levels of adhesion; dry and low as before ( $\mu=0.56$ and 0.072 respectively), with the addition of a very low condition $(\mu=0.038)$ which represents the state where even the use of defensive driving may not mitigate the operational risks experienced. The performance of the estimator in these conditions is shown in Figure 4.

It can be seen that as the adhesion level falls, the overall levels of creep force fall and the estimator correctly follows this magnitude change. The best match for force estimation is seen in the dry condition and the largest discrepancies exist at the very low adhesion condition. As the creep forces become small, the resultant wheelset deflections are not large enough to exceed the required breakout forces (i.e. the static friction part) of the suspension components. The linear estimation part of the Kalman-Bucy filter will still predict a proportional deflection in the suspension whereas the non-linear VAMPIRE ${ }^{\circledR}$ simulation these breakout forces will not be exceeded, creating a difference between estimated and actual sensor values (y 

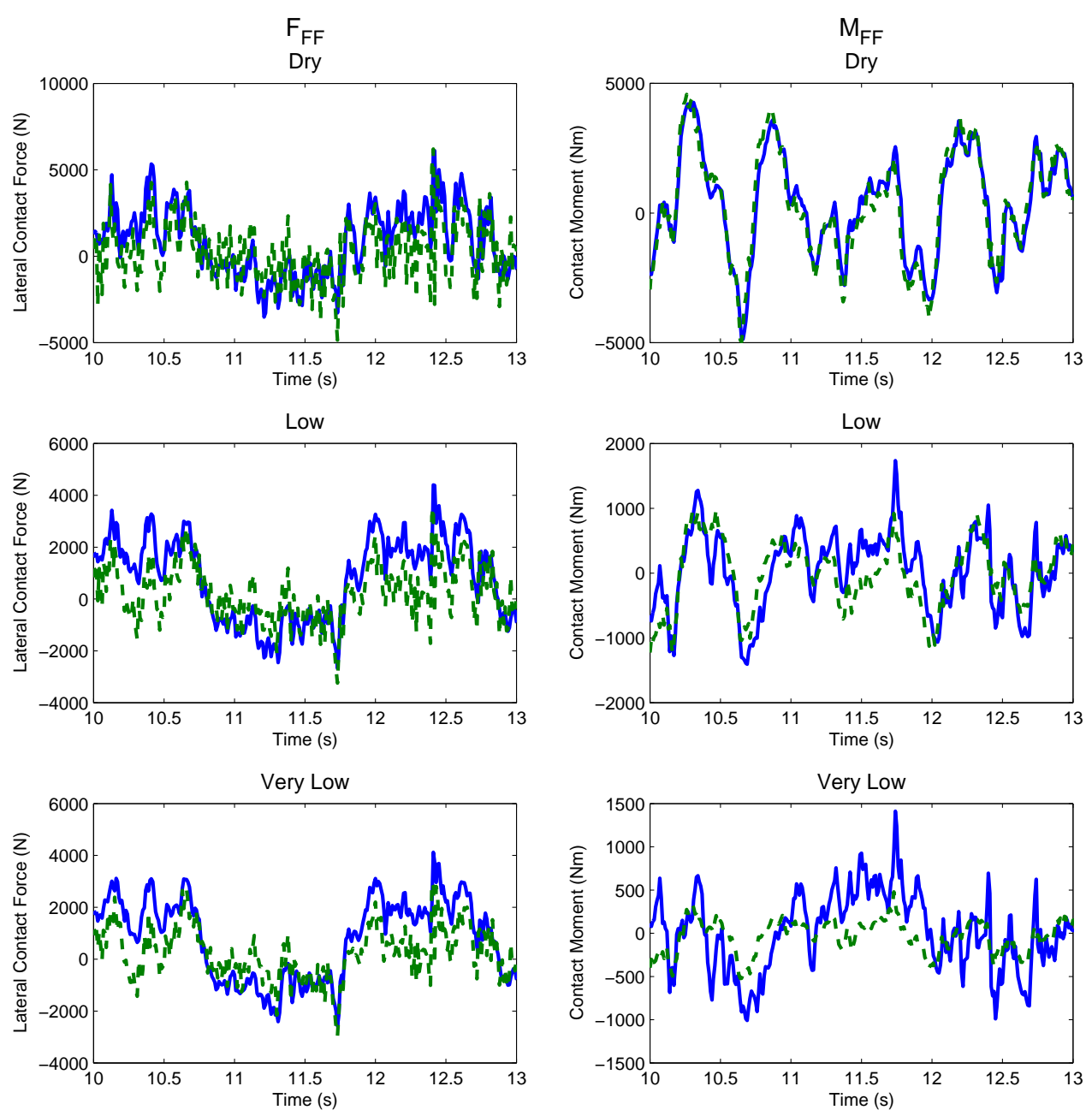

Fig. 4. Graphs showing the estimated creep forces (solid line) against the actual creep forces (dashed line) over three adhesion conditions.

and $\hat{\mathbf{y}}$ ). As a result, the contact information gets incorporated with the modelling discrepancies within the Kalman filter. 
However, the estimator successfully follows the overall fall in level of creep force, particularly with the reduction of creep moment with adhesion level. Figure 5 shows the performance of the estimator over the three adhesion levels when a five second moving RMS is applied to both the measured values and the estimated values.
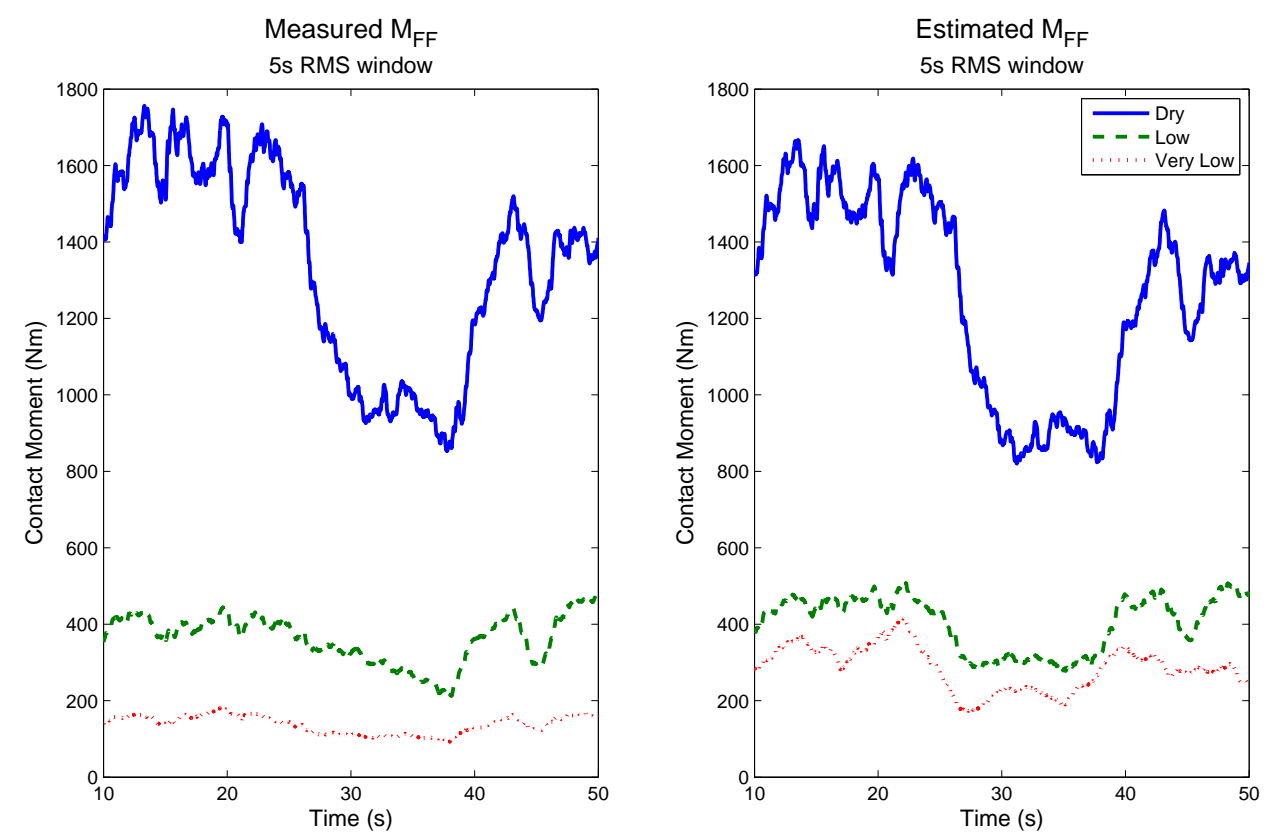

Fig. 5. Graphs comparing the measured creep moment to the estimated creep moment over three adhesion levels.

The variations observed in the RMS creep moment throughout an individual run is attributed to the naturally changing magnitude of irregularities experienced upon the track during that particular window. Normalising to track irregularities presents a challenge as they are difficult to measure onboard a standard railway vehicle and referring to stored information regarding track section presents a different set of operational difficulties. 
In order to provide normalisation to the track irregularity, tests were performed whereby the estimated creep force RMS values were normalised by each of the onboard measurable states (with a moving RMS taken over the same window) and assessed to observe performance. It was found that normalising by wheelset yaw acceleration provided the best results.

Because the conditions of each test run was known, the measured ratio of $M_{F F(R M S)}$ to $\ddot{\psi}_{F F(R M S)}$ when averaged over the whole test run can be used as a calibration value for level of adhesion. Therefore, if the ratio of $M_{F F(R M S)}$ to $\ddot{\psi}_{F F(R M S)}$ can be measured during a vehicle run the calibration values can be used to populate a linear interpolation table from which the current level of adhesion being experienced can be determined. The calibration data used is summarised in Table 2 .

\begin{tabular}{ccc} 
& \multicolumn{2}{c}{ Calibration Data } \\
\hline Condition & $\mu$ & $M_{F F(R M S)} / \tilde{\psi}_{F F(R M S)}$ \\
\hline \hline Dry & 0.56 & 3088 \\
Low & 0.072 & 1237 \\
Very Low & 0.038 & 900.6
\end{tabular}

Table 2. Calibration data for adhesion level with respect to Creep Moment/Yaw acceleration ratio

This method is validated on further VAMPIRE ${ }^{\circledR}$ simulations whereby a change in adhesion level part way through the test run is introduced. In these tests, a step change in adhesion is experienced after 30 seconds of travel. Figure 6 shows the actual adhesion level and estimated adhesion level of two test runs.

It can be seen that the estimated adhesion level correctly changes from high levels of adhesion to the lower levels. The discrepancy between actual and estimated values can be explained by the inability to scale accurately to track irregularity. It is also possible to see the time lag in the estimator response to the step change. This is due to the 5 second moving window over which an RMS is taken. Although the adhesion level is not accurately tracked, the overall change in operating conditions is identified. 


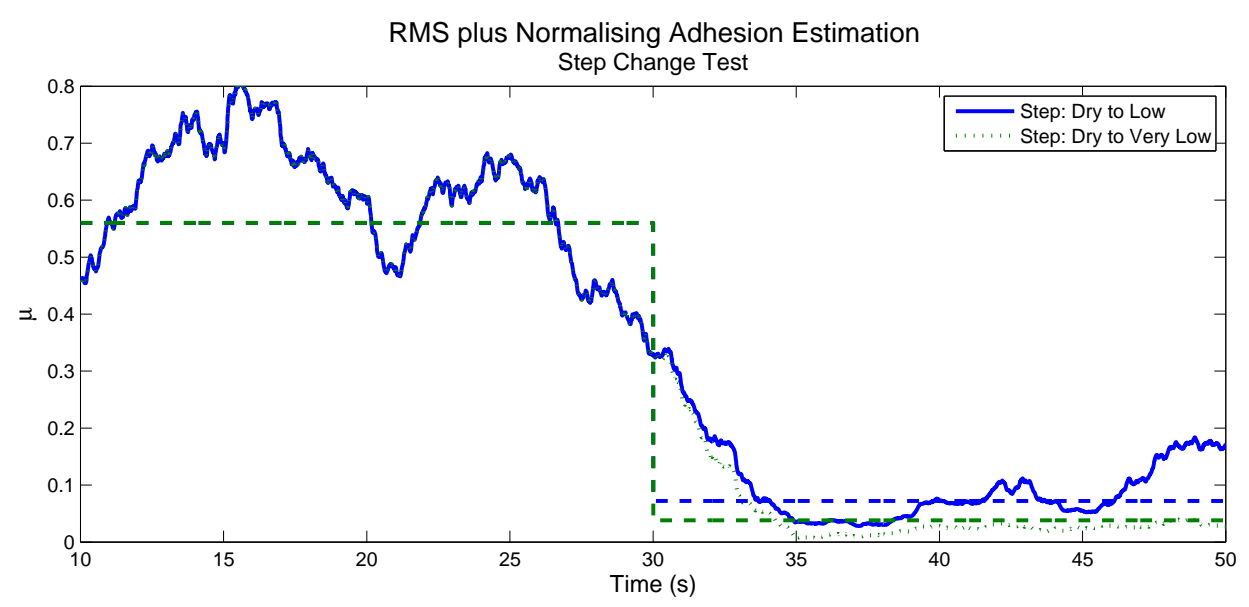

Fig. 6. Graph showing adhesion estimation during a step change in adhesion. The dashed line show the actual level of adhesion for each test.

\subsection{Further Work}

The model-based creep force estimation scheme used here has been shown to provide good estimations of the contact forces experienced in the wheel rail contact during MBS test scenarios.

Having obtained estimations of creep forces and moments, basic post processing has shown that it is possible to attain a reasonable approximation of adhesion, even with a coarse linear interpolation method based on only 3 points and an abstract method of normalising for track irregularity. Future studies may focus on the development of post-processing techniques to more accurately analyse the creep force data to better estimate adhesion.

A limitation of this technique is that, in its current form, the creep force estimator loses accuracy at low adhesion levels for suspension units with significant non-linearity in their components. In a case study with a British Rail (BR) Mk3 vehicle, fitted with a BT-10 bogie, it was found that the estimation method at low levels of adhesion was poor. The geometry of the suspension of this model is such that a linear 
plan view model does not sufficiently capture the dynamics of the vehicle, particularly when suspension components are operating with small deflections. This situation occurs either when there are small levels of track irregularities, or when the adhesion level is low. As such, vehicles with suspension dynamic response characteristics, such as the BR Mk3 vehicle, would require a more detailed mathematical model around which to form a filter.

\section{Direct Data Analysis}

The method presented in this section aims to identify any features or relationships between measurable data that can be used to signify a change in adhesion levels without the need for the complexity of developing a Newtonian based model. This approach has been used in identifying suspension system faults $(19 ; 20)$ by observing changes in the system dynamic interactions or by different frequency modes observed (21). All these methods rely on calibration against a baseline model to identify good operating conditions.

The vehicle used in this case study is a BR Mk3 vehicle. As mentioned in section 2, the non-linear characteristics inherent to this vehicle make it unsuitable for use with the model-based estimator technique presented due to the manner in which the suspension responds under very low adhesion levels for which there are correspondingly low levels of creep force.

After an initial search, the investigation followed two streams; relationships between leading and trailing bogie dynamic responses, and relationships between leading and trailing wheelset dynamic responses. 


\subsection{Leading and Trailing Wheelsets}

Under high adhesion conditions it is known that during quasi-static curving the forces and relative movements of the leading wheelset are significantly larger than for the trailing wheelset (15). It seems possible therefore that a comparison between leading and trailing wheelset dynamic data may provide a useful indication for low adhesion.

After an investigation on data produced by VAMPIRE ${ }^{\circledR}$ simulations for the Mk3 vehicle, the most significant dynamic quantity of interest was the yaw velocity comparison. Figure 7 shows the data recorded over a one second interval on a test track that contains lateral only disturbances. Figure 7 a shows this test under dry conditions and Figure $7 \mathrm{~b}$ shows this under very low conditions.

It can be seen that, at low adhesion conditions, the yaw velocity of the leading and trailing wheelsets, and bogie converge and begin to move more as a single unit. It is hypothesised that at low adhesion levels the creep forces are not of a significant magnitude to overcome the breakout forces of the stiff wheelset-bogie yaw connections and the secondary yaw friction levels inherent in parts of the BT-10 suspension design. The parts are therefore more likely to lock together and move as a unit, rather than display independent dynamic behaviour.

This analysis showed initially promising results as there is a noticeable trend between the level of adhesion and a correlation analysis of the dynamic responses. However, when an identical simulation test was performed with full track irregularities (i.e. vertical, cross level and gauge width irregularities), the results were less satisfactory.

This analysis shows that this does not yet appear to be a useful indicator for real-time adhesion detection. It has nevertheless revealed that the yaw motions of the two wheelsets in response to lateral irregularities 
a) Yaw Rate Comparison:

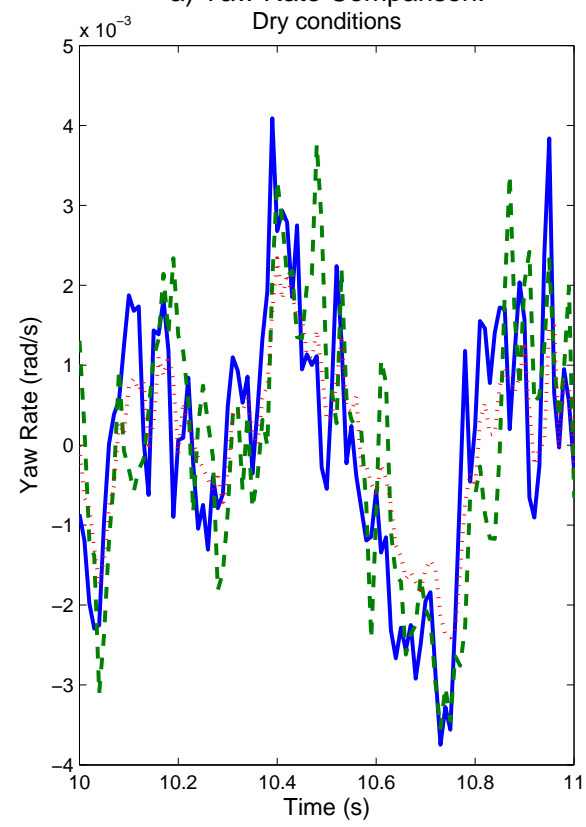

b) Yaw Rate Comparison

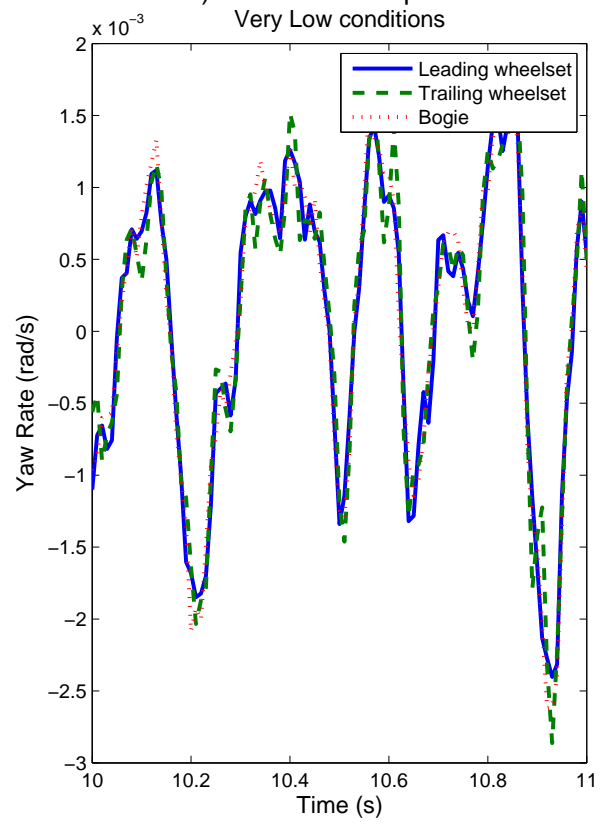

Fig. 7. Comparisons of bogie, leading wheelset and trailing wheelset yaw rate in a) Dry conditions and b) Very low adhesion conditions.

become increasingly similar as adhesion levels reduce. To pursue this investigation further, appropriate normalisation or scaling factors would require to be studied to attempt to clarify the correlation trends.

Initial testing on the modern DMU vehicle datasets show that relationship identified for the Mk3 vehicle does not remain true. It is apparent that the suspension features of the BT-10 are such that the breakout forces are not exceeded during the smaller impulses experienced with very low adhesion levels, whereas in the modern vehicle example they are. It remains to be seen if other dynamic measurements between wheelsets could be used in a similar ways to see if similar traits are present that could be exploited to detect low adhesion conditions. 


\subsection{Leading and Trailing Bogie Dynamics}

Similarly to the comparison of the leading and trailing wheelset dynamic responses, it was considered that a study to compare leading and trailing bogie dynamic responses may yield a useful indicator for levels of adhesion. Once again, this study focused on the BR Mk3 vehicle.

This investigation began by comparing the correlation of all dynamic variables of the leading and trailing bogies. The variables were compared when taken at the same instant in time, and when the leading data is delayed so the comparisons are made as the bogies travel over the same piece of track. All the possible dynamic measurements of the vehicle components were considered at different adhesion levels to see if there were observable differences in correlation as the friction changes.

This was performed using the Matlab function "crosscorr" (22) that provides an estimate of the correlation between two time series over a range of sample delays or 'lags'. The value for cross correlation is obtained by:

$$
\mathbf{r}_{x y}(k)=\frac{c_{x y}(k)}{s_{x} s_{y}}, k=0,1,2, \ldots(3.1)
$$

where $r_{x y}(k)$ is the estimate of the cross correlation, $c_{x y}(k)$ is the cross covariance function and $s_{x}$ and $s_{y}$ are the sample standard devations. Figure 8 show the cross-correlation analysis for the yaw dynamic quantities of the leading and trailing bogie.

It was found that the biggest change in correlation with adhesion was between the yaw angles of the two bogies. At high levels of adhesion, the correlation was found to be low $(r \approx 0.2)$ whereas at low levels of 

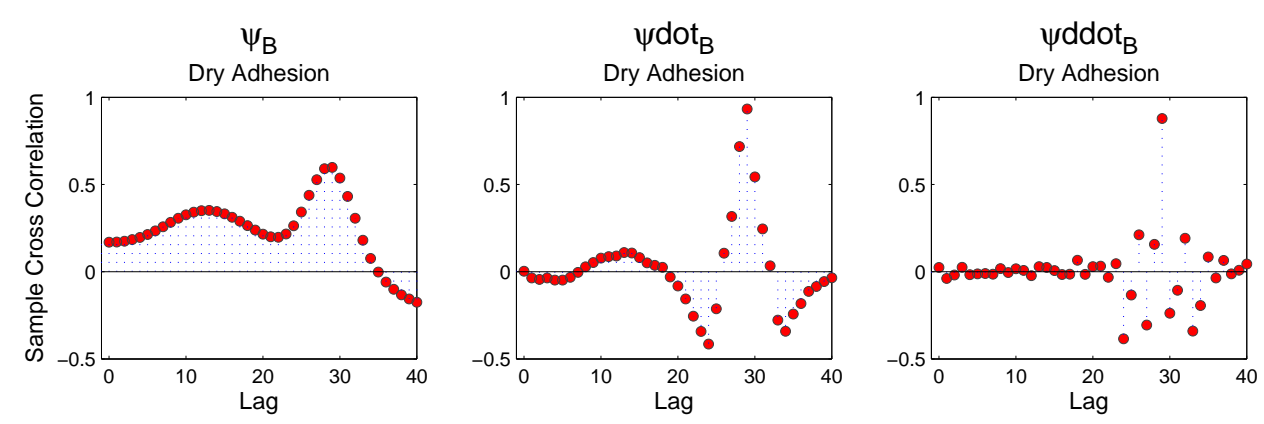

$\psi_{B}$
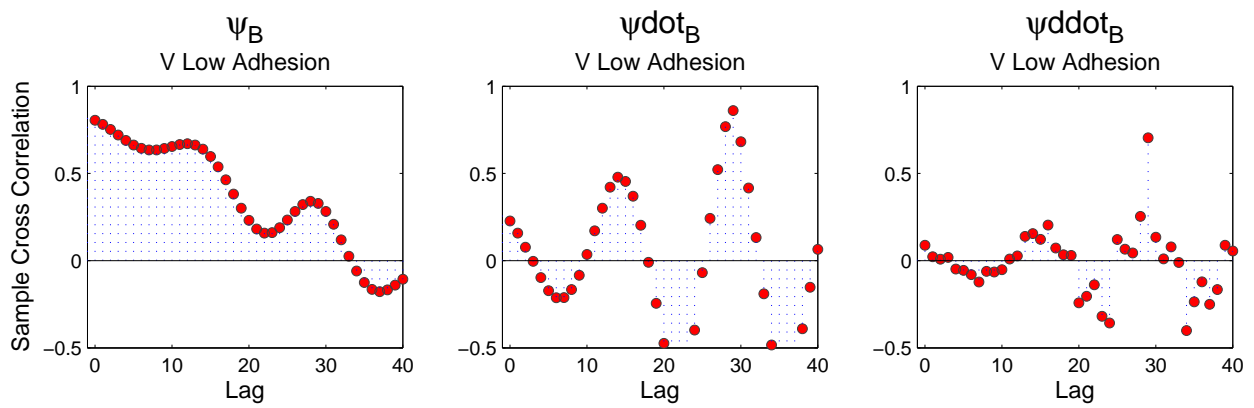

Fig. 8. Figure showing the cross-correlation analysis of leading and trialing bogie yaw position $(\psi)$, yaw velocity $(\dot{\psi})$ and yaw acceleration $(\ddot{\psi})$ in both dry and very low adhesion conditions.

adhesion the correlation was high $(r \approx 0.8)$. As with the results presented in section 3.1, it is thought that this relationship is once again a result of the levels of static friction within the Mk3 suspension components.

The trend between adhesion levels and agreement of bogie yaw position can therefore be exploited to produce an adhesion estimator. The scheme developed based on this relationship is shown in Figure 9. Here, the difference between leading and trailing bogie yaw angle is filtered by taking a RMS over a moving window. This value is then normalised for the variances in track irregularity by scaling with the RMS of the leading bogie yaw angle and then multiplied by the RMS of the leading wheelset lateral acceleration. 
Both these RMS are taken over the same moving window as the yaw angle. Testing concluded a 10 second moving window provided reasonable results.

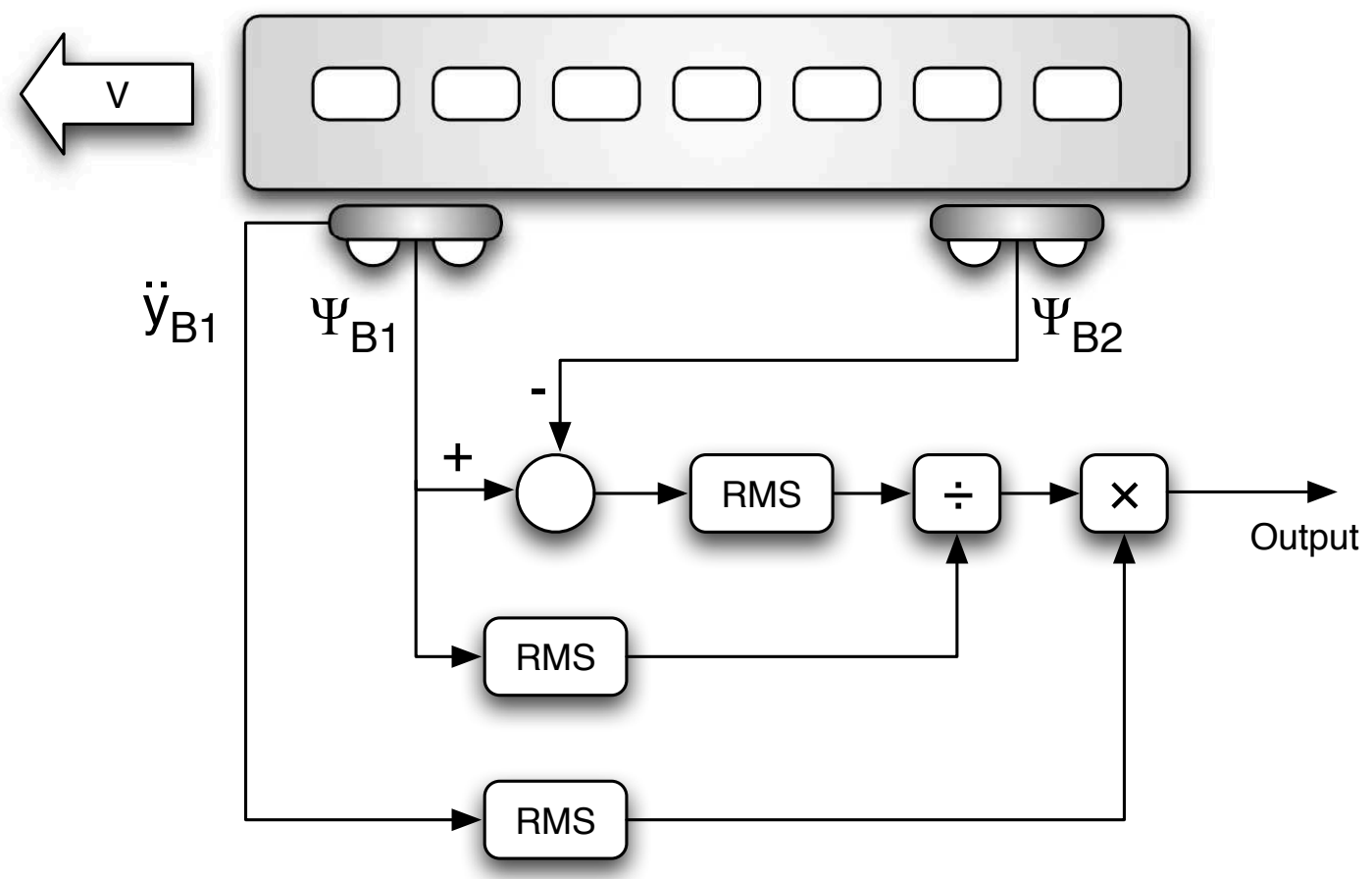

Fig. 9. Leading/Trailing bogie yaw comparison scheme where:

$\psi_{B 1}, \psi_{B 2}$ - bogie yaw position (leading and trailing) $\ddot{y}_{B 1}$ - leading bogie lateral acceleration

Four test runs utilising this system are summarised in Table 3. Each simulation consists of an instantaneous change in adhesion after 30 seconds in the direction highlighted in the third column of the table. The source data for these tests were generated by VAMPIRE ${ }^{\circledR}$ simulations of the Mk3 vehicle

The resultant scaled RMS of the difference between yaw angles derived from these test runs are shown in Figure 10. 


\begin{tabular}{ccc}
\multicolumn{3}{c}{ Test Run Summary } \\
\hline Run No. & Speekd (kph) & Adhesion Change \\
\hline \hline 1 & 200 & High to V Low \\
2 & 200 & V.Low to High \\
3 & 100 & High to V. Low \\
4 & 100 & V.Low to High \\
\hline
\end{tabular}

Table 3. Summary of conditions within the test runs. Adhesion change takes place after 30 s of travel

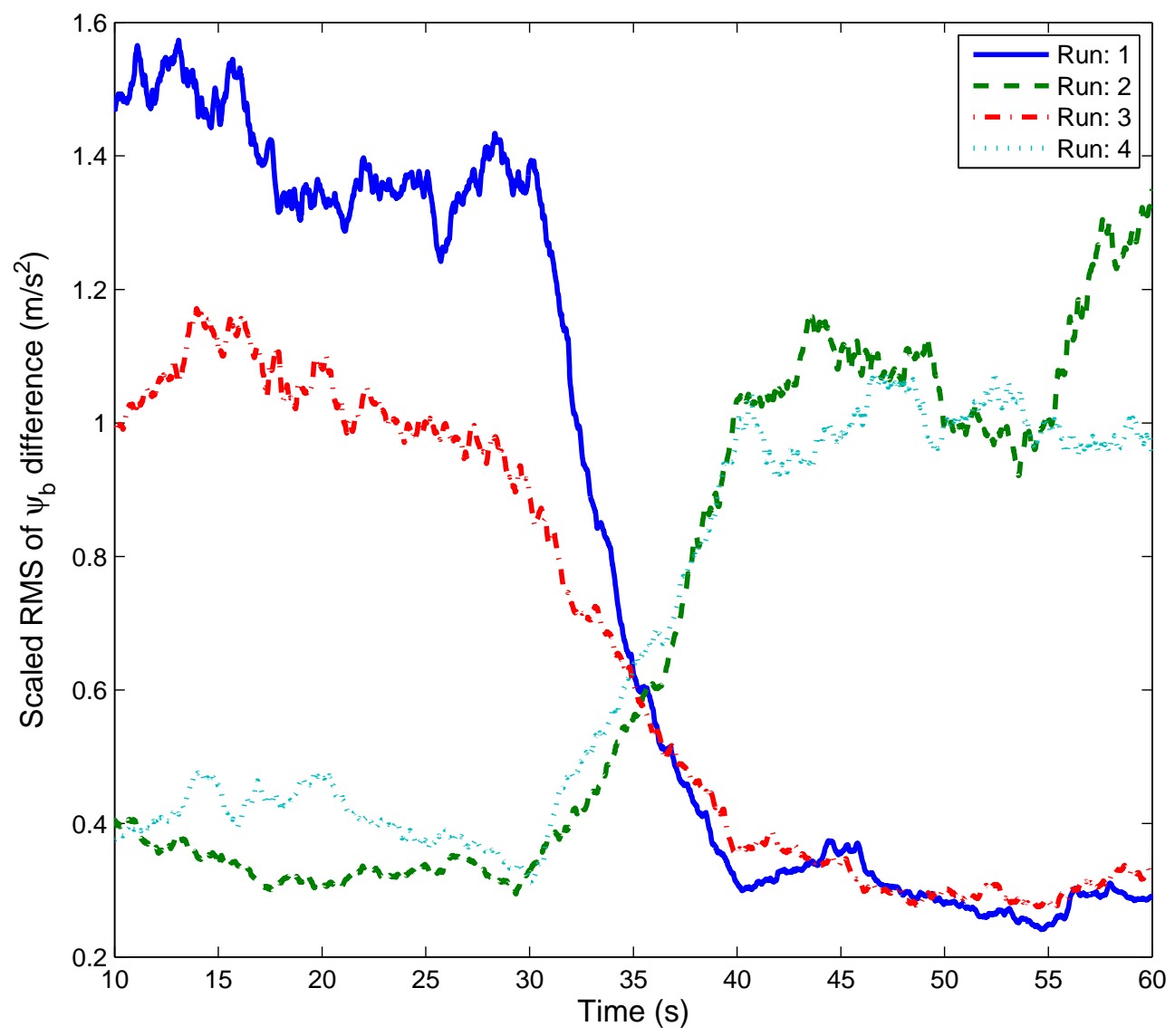

Fig. 10. Graph showing the scaled RMS of the difference between leading and trailing bogie yaw position for 4 test runs 
Figure 10 shows that there is a distinct change of the scaled RMS of the difference between yaw angles as adhesion levels change. Although this technique shows promise, there remains further investigations to confirm its viability as an adhesion detection method.

\subsection{Further Work}

One area of concern with this method is that the relationship identified relies on the specific suspension characteristics of the chosen case-study vehicle. An initial case study on the modern DMU vehicle data demonstrated that the relationship of bogie yaw angle agreement to adhesion level was not as prominent as shown for the Mk3 simulations, although the tehcnique was found to work with correlation analysis based upon different variables (not included in this article). In all likelihood, different trends between correlation patterns and adhesion levels will be observed for different suspension design philosophies.

Furthermore, although a distinct difference is observed between the two extreme conditions of high adhesion and very low adhesion, there is a requirement to distinguish between low and very low conditions. Although differences between these conditions can be observed within certain test runs, they are not as clear as the results shown above.

\section{Conclusions}

Two different methods have been demonstrated to estimate adhesion levels in the wheel/rail interface during the normal running of high fidelity model of a typical in-service rail vehicle. The model-based estimation technique demonstrates a good potential to provide accurate lateral force and yaw moment estimations on the modern style vehicle. Along with some basic post processing, a reasonable estimate of current adhesion level 
is observed, albeit with a small processing delay. This technique would benefit from further investigation into post-processing methods to identify more accurate and faster techniques of deriving adhesion.

Furthermore, the direct data analysis methods have provided some potentially useful markers for identifying low adhesion areas. As these methods approach the data directly, an accurate linear model of a complex, non-linear suspension model is not required. In the case of the Mk3 vehicle where the suspension is troublesome to model linearly, refinement of these techniques could provide a solution where a model-based estimator may not. Both this method and the model-based approach would benefit from testing on in-service vehicles to prove their validity away from simulation.

One of the main findings is that, without direct knowledge of track irregularity, it is not possible to obtain an instant value of adhesion based on creep force estimation. Operationally this means that the output of an adhesion estimator in this form will have limited use onboard a vehicle in terms of quickly advising a driver of conditions or scheduling braking in an onboard anti-slip controller. However, the output would be quick enough to provide a real-time overview of the current rail network state if enough vehicles were equipped with such a device.

\section{Acknowledgements}

This work was supported in part by RSSB under the project number T959.

Our acknowledgements go to the Technical Strategy Leadership Group (TSLG) for supporting this work, and RSSB for funding and managing the project and guiding it towards an appropriate solution to suit industrial needs. They also go to DeltaRail for providing simulation test data and assisting in the generation of linear model for use in the model based estimator. 
References

[1] Ward C, Goodall R, Dixon R. Creep Force Estimation at the Wheel-Rail interface. In: Proceedings of the 22nd International Symposium on Dynamics of Vehichles on Roads and Tracks. Machester: Â@ Manchester Metropolitan University; 2011.

[2] Ward CP, Goodall RM, Dixon R, Charles G. Condition Monitoring of Rail Vehicle Bogies. In: UKACC International Conference on Control. Coventry, UK: Â@ IFAC; 2010. .

[3] Ward CP, Goodall RM, Dixon R. Contact Force Estimation in the Railway Vehicle Wheel-Rail interface. In: Proceedings of the 18th IFAC World Congress. Milan, Italy: Â@ International Federation of Automatic Control; 2011. .

[4] Li P, Goodall R, Kadirkamanathan V. Parameter estimation of railway vehicle dynamic model using Rao-Blackwellised particle filter. In: Proceedings of the European Control Conference; 2003. p. 1-4.

[5] Li P, Goodall R, Weston P, Seng Ling C, Goodman C, Roberts C. Estimation of railway vehicle suspension parameters for condition monitoring. Control Engineering Practice. 2007 Jan;15(1):43-55. Available from: http://www.sciencedirect.com/science/article/pii/S0967066106000463.

[6] Fan H, Wei X, Jia L, Qin Y. Fault detection of railway vehicle suspension systems. In: Computer Science and Education (ICCSE), 2010 5th International Conference on; 2010. p. 1264-1269.

[7] Hayashi Y, Tsunashima H, Marumo Y. Fault Detection of Railway Vehicle Suspension Systems Using Multiple-Model Approach. Journal of Mechanical Systems for Transportation and Logistics. 2008;1(1):88-99.

[8] Tsunashima H, Mori H. Condition monitoring of railway vehicle suspension using adaptive multiple model approach; 2010 .

[9] Charles G, Goodall R, Dixon R. Model-based condition monitoring at the wheel-rail interface. Vehicle System Dynamics. 2008 Sep;46(sup1):415-430. 
[10] Bruni S, Goodall R, Mei TX, Tsunashima H. Control and monitoring for railway vehicle dynamics. Vehicle System Dynamics. 2007;45(7-8):743-779. Available from: http://www.tandfonline.com/doi/abs/10.1080/00423110701426690.

[11] Ward CP, Weston PF, Stewart EJC, Li H, Goodall RM, Roberts C, et al. Condition monitoring opportunities using vehicle-based sensors. Proceedings of the Institution of Mechanical Engineers, Part F: Journal of Rail and Rapid Transit. 2011;225(2):202-218.

[12] Gu RWN, Pislaru C, Ball A, F. Modern techniques for condition monitoring of railway vehicle dynamics. Journal of Physics: Conference Series. 2012;364(1):12016. Available from: http://stacks.iop.org/1742-6596/364/i=1/a=012016.

[13] DeltaRail. VAMPIRE; 2011. Available from: http://www.vampire-dynamics.com/.

[14] Kalman RE. A New Approach to Linear Filtering and Prediction Problems 1. 1960;82(Series D):35-45.

[15] Wickens A. Fundamentals of Rail Vehicle Dynamics. Taylor \& Francis; 2003.

[16] Garg VK, Dukkipati RV. Dynamics of Railway Vehicle Systems. Academic Press, INC. (London) LTD.; 1984.

[17] Kalker JJ. A Fast Algorithm for the Simplified Theory of Rolling Contact. Vehicle System Dynamics. 1982;11(1):1-13.

[18] Polach O. Creep forces in simulations of traction vehicles running on adhesion limit. Wear. 2005;258(7ât"8):992-1000.

[19] Mei TX, Ding XJ. A model-less technique for the fault detection of rail vehicle suspensions. Vehicle System Dynamics. 2008 Sep;46(sup1):277-287. Available from: http://dx.doi.org/10.1080/00423110801939154.

[20] Mei TX, Ding XJ. Condition monitoring of rail vehicle suspensions based on changes in system dynamic interactions. Vehicle System Dynamics. 2009 Aug;47(9):1167-1181. Available from: http://dx.doi.org/10.1080/00423110802553087.

[21] Oba T, Yamada K, Okada N, Tanifuji K. Condition Monitoring for Shinkansen Bogies Based on Vibration Analysis. Journal of Mechanical Systems for Transportation and Logistics. 2009;2(2):133-144. 
[22] MathWorks. Matlab R2013a Documentation Centre, crosscorr; 2013 . Available from: http://www.mathworks.co.uk/help/econ/crosscorr.html. 